\title{
Consumo do Cigarro Eletrónico em contexto Laboral: proibir, tolerar ou incentivar?
}

www.rpso.pt/consumo-do-cigarro-eletronico-em-contexto-laboral-proibir-tolerar-ou-incentivar/

September 29, 2016

\section{E-CIGARETTE CONSUMPTION IN WORK CONTEXT: FORBID, TOLERATE OU ENCOURAGE?}

TIPO DE ARTIGO: Revisão Bibliográfica Integrativa AUTORES: Santos M(1), Almeida A(2).

\section{RESUMO}

Introdução

A legislação progressivamente mais rigorosa em relação ao tabagismo fez com que os fumadores fumassem menos ou até passassem a ser não-fumadores. Em função disto, as tabaqueiras ficaram mais aliciadas a explorar outras formas de consumir nicotina. Entre todos os métodos disponíveis, o cigarro eletrónico é considerado o mais agradável. Para além disso, a maioria dos fumadores quer deixar de fumar ou, pelo menos, diminuir o consumo.

\section{Metodologia}

Foi realizada uma pesquisa em junho de 2016 nas bases de dados "CINALH plus with full text, Medline with full text, Cochrane Central Register of Controlled Trials, Cochrane Database of Systematic Reviews, Cochrane Methodology Register, Nursing and Allied Health Collection: comprehensive, MedicLatina e Academic Search Complete". Utilizando as expressões-chave "electronic cigarette" e "e-cigarette" foram obtidos 248 e 287 artigos, respetivamente, com os critérios de publicação igual ou superior ao ano de 2009 e acesso a texto completo; em função da língua original (portuguesa, inglesa ou espanhola) e da pertinência para o objetivo desta revisão, foram selecionados 64 artigos.

Conteúdo

Com uma frequência cada vez maior, os fumadores questionam os profissionais de saúde relativamente ao cigarro eletrónico; uns porque já o consomem, outros porque estão a pensar fazê-lo, inclusive em ambiente laboral. É necessário, por isso, estar a par das conclusões dos estudos já elaborados sobre o tema.

Conclusões

Globalmente considera-se que a publicidade é enganosa, ou seja, o ato não é isento de riscos, nem está provado por estudos robustos que contribua, neste momento, para a cessação do consumo da nicotina. Contudo, simultaneamente, também parece claro que os agentes químicos envolvidos estão em muito menor número e concentração que no cigarro convencional, pelo que parece ser uma alternativa menos tóxica de consumir nicotina. No entanto, passar tal mensagem também pode contribuir para a diminuição da motivação para realizar a cessação tabágica ou pode até aliciar não-tabagistas ao início do consumo, comprometendo os ganhos obtidos na luta contra o tabaco das últimas décadas. 
Logo, o seu consumo em meio laboral deve ser desaconselhado e as restrições existentes para o cigarro convencional também devem ser aplicadas ao cigarro eletrónico.

Palavras- chave: cigarros eletrónicos, saúde do trabalhador, medicina do trabalho.

\section{ABSTRACT}

Introduction

The legislation regarding smoking caused smokers to smoke less or some even tried to became non-smoker. Because of this, the tobacco companies were more motivated to explore other ways of consuming nicotine. Among all the methods available, the electronic cigarette is considered the most pleasant. Furthermore, most smokers want to quit smoking or at least reduce consumption.

Methodology

A survey was done in June 2016 in the databases "CINALH plus with full text, Medline with full text, Cochrane Central Register of Controlled Trials, Cochrane Database of Systematic Reviews, Cochrane Methodology Register, Nursing and Allied Health Collection: comprehensive, MedicLatina and Academic Search Complete". Using the key words "electronic cigarette" and "e-cigarette" were obtained 248 and 287 articles, respectively, with the publication criteria greater than or equal to 2009 and access to full text; depending on the original language (Portuguese, English or Spanish) and their relevance to the purpose of this review, we selected 64 articles.

Content

With increasing frequency, smokers question the health professionals about electronic cigarette; some because they already consume, others because they are thinking on doing so, including in the work environment. We must therefore be aware of the findings of studies already done on the subject.

Conclusions

It is considered that the advertising is misleading: the act is not absent from risks, nor is proved by robust studies to contribute for the cessation of nicotine consumption. However, at the same time, it also seems clear that the chemicals involved are fewer and present in lower concentration than the conventional cigarette- so it seems to be a less toxic alternative to consume nicotine. However, to pass such a message can also contribute to decreased motivation to make smoking cessation or may even motive non-smokers to begin consumption, undermining the gains made in the fight against tobacco in decades. Therefore, its use in the work environment should not be recommended and restrictions for conventional cigarettes should also be applied to the electronic cigarette.

Keys-words: electronic cigarettes, occupational health, occupational medicine.

\section{INTRODUÇÃO}

A legislação progressivamente mais rigorosa em relação ao tabagismo fez com que os fumadores fumassem menos ou até passassem a ser não-fumadores. Em função disto, as tabaqueiras ficaram mais aliciadas a explorar outras formas de consumir nicotina ${ }^{1,2}$. Entre todos os métodos disponíveis, o cigarro eletrónico (CE) é considerado o mais agradável. ${ }^{1}$ Pois se o consumo do cigarro convencional ou clássico (CC) foi diminuindo ao longo dos anos $^{3,4}$, o inverso ocorreu para os novos produtos de tabaco entretanto desenvolvidos, ainda que o CC continue a ser o produto de tabaco mais utilizado ${ }^{3}$. Por exemplo, estima-se que cerca de $21 \%$ dos adultos dos EUA consuma alguma forma de tabaco, pelo menos, 
esporadicamente. Neste país o consumo é mais frequente no sexo masculino e nas zonas mais rurais ${ }^{4}$. Calcula-se também que cerca de 5,6 milhões de americanos com menos de 18 anos irão morrer prematuramente devido a doenças associadas ao tabaco. Também nesse ano (2015) estimou-se que cerca de 4,7 milhões de alunos do secundário e preparatório fossem consumidores usuais de algum produto com tabaco e que 2,3 milhões consumissem dois ou mais produtos. A exposição à nicotina durante a adolescência poderá potenciar o surgimento da dependência. De forma mais específica, os autores quantificaram $25,3 \%$ dos estudantes do ensino secundário usava algum produto com tabaco e 13\% referia utilizar dois diferentes; para o ensino preparatório os valores registados fora, 7,4 e 3,3\%, respetivamente. De 2011 a 2015, por exemplo, entre todos os estudantes, a prevalência de consumo do CE variou de 1,5 para $16 \%{ }^{5}$.

Existe um público-alvo diversificado para o consumo de produtos alternativos de nicotina: os que querem parar de fumar o $\mathrm{CC}$, os que querem continuar a fumar em locais públicos, os que querem fumar com menos toxicidade e/ ou os que querem gastar menos ${ }^{6-9} \mathrm{e} / \mathrm{ou}$ diminuir a toxicidade para os conviventes ${ }^{9}$.

A maioria dos fumadores quer deixar de fumar ou, pelo menos, diminuir o consumo e, geralmente, já realizou várias tentativas para parar que, globalmente, falham (quer devido à dependência direta da nicotina, quer pela ausência dos comportamentos e sensações associados ao ato de fumar). Estimou-se que, sem qualquer ajuda, apenas $5 \%$ se mantinha abstinente passado um ano e que cerca de metade dos que tentam recaia dentro da primeira semana. As técnicas comportamentais e farmacológicas potenciam os resultados mas, ainda assim, estes são modestos ${ }^{10}$. Estudos defendem que a terapia de reposição de nicotina (TRN) quase duplica a taxa de cessação a curto prazo mas, no final de 12 meses, apenas menos de $20 \%$ se mantém abstinente ${ }^{11}$. Outros autores, por sua vez, estimaram que somente 3 a $5 \%$ se mantém abstinente passados seis meses ${ }^{12}$. Alguns artigos ainda quantificaram que a TRN tem uma eficácia de $7 \%$ e que algumas medicações orais terão $20 \%{ }^{9}$.

A legislação ou a ausência da mesma sobre o CE difere muito de país para país. Contudo, na Europa, a Diretiva 2014/40/EU englobou o CE na lista de produtos associados ao consumo de tabaco estabelecendo que este deixaria de poder ser consumido "em recintos fechados destinados a utilização coletiva, nos recintos de diversão, nos casinos, bingos, salas de jogo e outro tipo de recintos destinados a espetáculos de natureza não artística ou em qualquer outro lugar onde, por determinação da gerência, da administração ou de outra legislação aplicável, designadamente em matéria de prevenção de riscos ocupacionais, se proíba fumar"; esta diretiva foi retificada para Portugal através da Lei 109/2015, de 26 de Agosto. Nos Estados Unidos desde 2009 que a FDA (Food and Drug administration) classifica o CE como um dos produtos do tabaco e não como terapêutica para a cessação tabágica ${ }^{1,13,14}$, tendo regulado o seu consumo em agosto de 2016 . Não obstante a sua regulamentação o consumo de CE tem vindo a aumentar significativamente nos últimos anos.

Apesar das dúvidas de segurança e da ausência de controlo de qualidade, o produto entrou muito rapidamente no mercado e está em constante aperfeiçoamento, apesar de em Portugal ter havido um decréscimo da procura, fruto do aumento de impostos instituído em 2015 sobre estes produtos (Lei do orçamento de estado para 2015). No entanto, parte dos consumidores deste produto poderão querer usufruir do mesmo em ambiente laboral, pelo que se pode colocar em causa a saúde e segurança para os restantes trabalhadores. 
Foi realizada uma pesquisa em junho de 2016 nas bases de dados "CINALH plus with full text, Medline with full text, Cochrane Central Register of Controlled Trials, Cochrane Database of Systematic Reviews, Cochrane Methodology Register, Nursing and Allied Health Collection: comprehensive, MedicLatina e Academic Search Complete". Utilizando as expressões-chave "electronic cigarette" e "e-cigarette" foram obtidos 248 e 287 artigos, respetivamente, com os critérios de publicação igual ou superior ao ano de 2009 e acesso a texto completo; em função da língua original (portuguesa, inglesa ou espanhola) e da pertinência para o objetivo desta revisão, foram selecionados 58 e 61 artigos após a leitura do resumo e 30 e 34 trabalhos, respetivamente, após a leitura do texto na íntegra. No fluxograma podem ser consultados dados relativos à pesquisa efetuada.

\section{CONTEÚDO}

Constituintes do cigarro eletrónico e modo de funcionamento

Os cigarros eletrónicos são dispositivos com bateria incorporada, que administram nicotina ${ }^{6-9,11-37}$, com ou sem aroma ${ }^{7-9,11,14-16,19-21,23,25,27-34,36-41 ~(f r u t a, ~ m e n t a, ~ c h o c o l a t e, ~}$ café, álcool, coca-cola, waffers, baunilha, chiclet ou até tabaco...- alguns autores afirmam que existem cerca de 7000 opções ${ }^{21}$ ) e outros agentes químicos, via inalação do aerossol $18,23,24,35,38$, sem queimar as folhas de abaco ${ }^{35}$. Além da bateria, existem ainda o bocal, cartucho e o vaporizador ${ }^{11,20,27,29,31-33,40,41}$. Estes dispositivos também podem ser designados por sistemas eletrónicos de libertação de nicotina (ENDS) 11,12,17,40,42. Têm uma aparência semelhante ao cigarro clássico ${ }^{11,20,32-34,38}$ e algumas marcas até têm uma luz na ponta, para mimetizar com rigor a versão original do produto ${ }^{38,40,41}$. Alguns são ainda recobertos por papel, para proporcionar a mesma sensação tátil ${ }^{40}$. De realçar que os produtos com aromas são sobretudo consumidos pelos mais jovens e iniciados no tabagismo ${ }^{19,29,43}$, pelo que a adição de aromas é criticada por várias instituições. $69 \%$ dos consumidores do CE (num estudo com quase 5000 consumidores) afirmaram trocar frequentemente de aroma; às vezes, até durante o mesmo dia ${ }^{29}$. A maioria afirmou que tal era fundamental para a redução ou cessação tabágica. Globalmente o aroma preferido era o frutal, ainda que no início tal papel estivesse entregue ao aroma a tabaco ${ }^{29,44}$ ou ao mentol $^{33}$, sobretudo em afro-americanos ${ }^{44}$. Pensa-se que no início o indivíduo quer uma experiência o mais parecida com o CC mas, à medida que o olfato melhora e/ ou interrompe o CC, prefere outro aroma ${ }^{29}$. Após obter alguma experiência, alguns chegam mesmo a adquirir dispositivos alterados em relação ao CE usual- estes já não se assemelham tanto ao CC, mas a bateria tem geralmente voltagem superior, o que permite vaporizar mais líquido. Para além disso, surgiram também modelos cada vez mais esteticamente estilizados. A curva de aprendizagem irá posteriormente determinar a escolha do dispositivo e líquido; até a perceção de ser necessário trocar de bateria e/ou cartucho de líquido se aperfeiçoa com a experiência ${ }^{33,45}$; após algum treino um consumidor de CE pode obter níveis de nicotina equivalentes ao $\mathrm{CC}^{45}$.

Para além do aroma tornar o produto mais apelativo, sobretudo para os mais jovens, alguns investigadores também defendem que o agente químico que justifica o aroma pode apresentar toxicidade suplementar; poderá ainda interagir com a nicotina (sobretudo o mentol, baunilha e o aroma frutal) ou conter impurezas ${ }^{44}$.

O produto foi inicialmente desenvolvido por uma empresa chinesa em 20041,11,17,18; 
inicialmente designada por Ruyan Group e, posteriormente, Dragonite ${ }^{17,18}$ ou em $2003^{8,27,35}$ pela empresa Hon Lik ${ }^{46}$, consoante os artigos consultados. Contudo, ao longo dos anos, muitas outras entidades desenvolveram e aperfeiçoaram inúmeros modelos11,46. Presentemente acredita-se que existem mais de $100^{18}$ a $400^{20,21}$ marcas, progressivamente aperfeiçoadas, sobretudo a nível da libertação de nicotina ${ }^{11,18,46}$. A solução de nicotina é vaporizada após acionar um botão ${ }^{17,18,47}$, por aquecimento $17,38,42,46$, entrando o vapor pela boca ${ }^{6,38,42,47}$ (mas a temperaturas menores que o $\mathrm{CC}$ ) ${ }^{47}$ ou através de um sensor de fluxo que, quando ativado, aquece a solução de nicotina ${ }^{38,46,48}$. A eficácia do processo depende da concentração de nicotina no líquido, da qualidade da vaporização e da biodisponibilidade da nicotina no aerossol ${ }^{30,38}$. Enquanto o CC está continuamente a arder a uma temperatura relativamente constante, no CE existem ciclos de aquecimento e arrefecimento; quando se ativa o mecanismo, o líquido é levado até o ponto de ebulição, de forma a este se transformar em vapor. Se se interromperem os puffs, não é transmitida energia à resistência, o que leva à diminuição da temperatura ${ }^{39}$. Contudo, a duração média dos puffs com o CE é cerca do dobro da que existe com o $\mathrm{CC}^{30,39}$, o que diminui o volume exalado; aliás, se se reter a respiração por alguns segundos, é possível não se exalar qualquer vapor; os consumidores que queiram mimetizar com rigor o ato de fumar CCs podem, por isso, fazer puffs mais curtos. O ritmo da evaporação e o controlo térmico dependem da duração dos puffs; se o dispositivo for ativado antes de a temperatura diminuir, pode entrar em sobreaquecimento, originando um puff "seco", ou seja, proporciona uma sensação desagradável de queimadura. Diferentes modelos têm mecanismos diversos de arrefecimento ${ }^{39}$.

Os principais agentes químicos identificados são o propilenoglicol e o glicerol/ glicerina69,19,21,25,27-32,36,38,39; ou seja, os produtos são tóxicos mas encontram-se em menor número e concentração que no CC13. A primeira destas substâncias pode causar alguma irritação das vias respiratórias superiores ${ }^{19,25}$ e olhos; a exposição prolongada ou repetida em contexto ocupacional a estes produtos associa-se a alterações esplénicas, do sistema nervoso central e comportamentais. O propilenoglicol pode dar origem ao formaldeido ${ }^{30} \mathrm{e}$ óxido de propileno, que é classificado pela IARC (Agência Internacional de Pesquisa para o Cancro) como carcinogénico, classe $2 \mathrm{~B}^{19}$. Por sua vez, o glicerol pode formar acroleína ${ }^{19,49}$, que também é irritante das vias respiratórias superiores ${ }^{19}$.

O vapor exalado contém nicotina, propilenoglicol, dietilenoglicol, formaldeído, propanolol, acetona, acetaldeído, isopreno, ácido acético e alguns metais $6,21,25,46$; outros autores especificam produtos como cálcio, alumínio, magnésio, prata, cobre, níquel, crómio, ferro, manganês, fibras de vidro e silício ${ }^{50}$-mas não existe combustão $0^{6,8,25,27,30,32,39,42}$ ou fumo6,8- apenas ocorre evaporação ("vaping") ${ }^{25}$, não havendo por isso emissão de monóxido de carbono ${ }^{19}$. Algumas investigações destacam também a presença de chumbo e cádmio associados a alterações hematológicas, renais, hepáticas, ortopédicas e vasculares. De salientar que há quem defenda que a maioria dos agentes químicos do CE entra no organismo através da mucosa oral e não pela via respiratória21. Contudo, é de salientar que o propilenoglicol existe, por exemplo, nos fumos das discotecas ou espetáculos, sendo ainda usado como aditivo alimentar e na indústria farmacêutica. Aliás, alguns autores consideram que a náusea, cefaleia e tonturas podem se associar mais à nicotina ${ }^{12,51}$.

A exposição às nitrosaminas pode ser estimada em função da concentração destas no líquido, sem necessidade de analisar o aerossol. Estas substâncias são cancerígenas mas 
a sua concentração no líquido é diminuta e não diferente da existente dos produtos da TRN; elas derivam de produtos existentes nas folhas do tabaco trabalhado (nas folhas verdes elas não são encontradas) ou após pirólise ${ }^{52}$.

Quanto à concentração de diversos materiais associados ao CE, alguns autores concluíram que a exposição não é significativa, comparando com o $\mathrm{CC}$, mesmo com uso intenso do CE (dobro da média estimada). Ainda assim, segundo estes autores, os mais relevantes foram o cádmio, o ferro e o níquel ${ }^{53}$.

Algumas marcas disponibilizam cartuchos sem nicotina ${ }^{18,29}$; contudo um estudo quantificou que nem $3 \%$ dos utilizadores escolhiam essa opção ${ }^{29}$. A generalidade das marcas classifica o teor de nicotina nos níveis extraforte, forte, médio, suave e muito suave ${ }^{38}$. Contudo, alguns dos produtos rotulados como sem nicotina, continham alguma ${ }^{19,38}$. Na generalidade dos casos, a nicotina varia entre os zero e os $20 \mathrm{mg} / \mathrm{ml} 25$. Quantificou-se que cada puff terá entre 0 a $35 \mathrm{mg}$ de nicotina ${ }^{21,34}$; assumindo o valor médio de 30 , seriam precisos cerca de $10^{34}$ a 30 puffs para obter uma dose equivalente à geralmente obtida por um CC. Para além disso, não há uma correlação direta entre a concentração de nicotina no cartucho e a que existe em cada puff, já que também têm de ser consideradas questões mecânicas do dispositivo ${ }^{19}$.

Alguns autores dividem o CE em três categorias:"cigalikes", ou seja, dispositivos com o mesmo tamanho e forma que o CC, descartáveis ou não, carregáveis ou não; "ego's" ou canetas vaporizadoras; estes geralmente são maiores e mais alongados e têm uma bateria mais potente, sendo geralmente possível o carregamento e "mods", que são os dispositivos com maiores dimensões e mais dispendiosos, aceitando geralmente a possibilidade de recarga.

A qualidade do ar onde se fumam cigarros eletrónicos depende da ventilação, área, tempo de consumo e de consumidores, diferentes composições dos cartuchos/ marcas, idade do dispositivo, duração e intervalo entre os puffs ${ }^{25}$.

\section{Marketing}

Desde 1971, na América do Norte, que a publicidade televisiva ao tabaco está proibida, devido à influência exercida, sobretudo nos mais jovens; em 1986 a proibição alastrou para a rádio também. Até aí as tabaqueiras ocupavam cerca de $10 \%$ da publicidade televisiva e era extremamente comum verem-se filmes (ou até desenhos animados) com indivíduos a fumar. Por vezes, programas de maior audiência eram patrocinados justamente por estes produtos. A aceitabilidade da população perante a publicidade começou a mudar quando se divulgaram os resultados dos primeiros estudos mais robustos, associados às consequências do tabagismo e quando se salientou que eram justamente as crianças e os jovens os mais suscetíveis de serem seduzidos pela publicidade; aliás, poderia mesmo simbolizar a entrada no mundo dos adultos. Para além disso, genericamente, também se considera que quanto mais precoce é o início do consumo, maior é a probabilidade de o indivíduo se transformar em fumador crónico. Pensa-se também que as crianças tenham maior dificuldade em distinguir o CE do $\mathrm{CC}^{40}$.

Na publicidade (nos EUA) a este produto são usadas imagens de figuras públicas desde 2009. Aliás, em quase metade das campanhas analisadas desde 2012 (22 em 59), era a personagem de um médico que apresentava o produto. Dentro destas, em $95 \%$ delas afirmava-se que o produto era mais saudável, 93\% salientava ser mais económico, 95\% mais limpo, 88\% alegava que se podia consumir em qualquer local, $71 \%$ permitia contornar a legislação antitabágica, $76 \%$ que não produzia fumo (só se libertava vapor de água 
inofensivo) e $73 \%$ das campanhas diziam que se tratava de uma modernidade. $64 \%$ dos sites apresentavam supostos testemunhos diretos de sucesso19. Neste país a publicidade também menciona menor probabilidade de patologia cardíaca e pulmonar, bem como menor incidência de nascimento de bebés com baixopeso ${ }^{21}$.

No Reino Unido, por sua vez, as campanhas publicitárias avaliadas até 2012, salientavam sobretudo que o CE era uma escolha mais saudável, possibilitando contornar as restrições tabágicas legislativas, ser usado por figuras públicas e ter preço inferior. Por vezes até surgiram comparações humorísticas com tentativas prévias fracassadas de TRN ${ }^{19}$.

Uma investigação que pretendeu avaliar as reações desencadeadas pela publicidade ao $\mathrm{CE}$, quantificou que $76 \%$ dos consumidores de CC ficavam com vontade de fumar CCs, $74 \%$ pensou em parar de fumar, $66 \%$ ponderou a possibilidade de experimentar o CE no futuro mas $34 \%$ dos que já consumiam o CE ficavam com vontade de fumar o CC. Entre géneros, o sexo feminino parece estar mais disponível para experimentar o CE. Dentro dos consumidores, a maioria fuma simultaneamente os dois tipos de cigarros, seguido de um grupo de ex-fumadores de CC, sendo menor o grupo que nunca fumou o $\mathrm{CC}^{19}$.

Um estudo quantificou que consumidores de CC têm maior apetência para experimentar o CE, ou seja, 70,2 versus 7,6 dos indivíduos que nunca fumaram e de forma estatisticamente significativa. Estes últimos afirmam que os principais motivos para utilizar o CE são a curiosidade e o facto de conviventes também o fazerem, de forma mais intensa em adolescentes. A principal motivação para parar com o CC é o receio de complicações médicas ${ }^{54}$.

Algumas marcas de CE fazem publicidade via Twitter. Uma vez que os recetores das mesmas podem reencaminhar as mensagens, é possível que o conteúdo chegue a um público mais vasto, sem controlo de idade, sobretudo quando estão associados aromas55. Outro fórum com popularidade mundial é o Reddit e nele também se encontra publicidade ao CE. Também são mencionados o YouTube (com vídeos divulgadores) e o facebook. $O$ Reddit foi criado em 2005 e fornece notícias e entretenimento; os usuários votam e elaboram rankings dos conteúdos e dos comentários, de forma anónima ${ }^{44}$.

Uso do CE para cessação tabágica ou para diminuição do consumo do CC

Alguns estudos afirmam que a maioria dos consumidores do CE fazem-no com o objetivo inicial de parar de fumar ${ }^{6}$. Na opinião de alguns autores este consumo consegue atenuar a abstinência da nicotina $24,32,45,56$, em ambos os géneros ${ }^{32}$ (com particular enfâse na capacidade de concentração ${ }^{32,56}$ e habilidades cognitivas ${ }^{56}$ - como a memória, bem como na ansiedade, irritabilidade e depressão ${ }^{32}$ ), pelo que inferiram que este utensílio é capaz de proporcionar uma quantidade razoável de nicotina ${ }^{56}$. Ao contrário da FDA, a instituição "Medicines and Health Care Products" não descarta a possibilidade que, no futuro, se considere o CE como terapêutica para a cessação, sujeito a prescrição ${ }^{24}$.. No entanto, um estudo quantificou que a concentração de nicotina nos cartuchos, para permitir uma assimilação adequada ${ }^{39}$, deverá ser na ordem dos 18 a $20 \mathrm{mg} / \mathrm{ml}$ - com 16 mg a abstinência é menor mas já não estatisticamente significativa ${ }^{32}$; no entanto, a concentração média fornecida pela generalidade dos produtos constituintes da TRN clássica é cinco vezes superior ${ }^{39}$. Curiosamente, outros investigadores defendem o oposto e afirmam que a libertação de nicotina pelos produtos de TRN é inferior à do CE. O preço do kit inicial é semelhante mas, futuramente, o consumidor apenas tem de adquirir as recargas, pelo que se torna uma opção muito mais económica ${ }^{33}$. Para além disso, alguns 
investigadores consideram que o CE de 16 mg é mais eficaz que a TRN ${ }^{32}$.

Alguns profissionais de saúde defendem que a informação a passar aos fumadores deverá clarificar que, na realidade, neste momento, não se sabe quanto eficazes ou seguros são os CEs mas, simultaneamente, está claro que são menos tóxicos que o $\mathrm{CC}^{24,57}$; não rejeitando a possibilidade que, um dia, este dispositivo possa ser usado na cessação tabágica, até em conjunto com a TRN convencional e/ ou outros produtos (com o Champix®, por exemplo). Apesar de alguns assumirem a atitude pragmática de que o que quer que possa eventualmente ajudar na cessação tabágica, merece uma hipótese de aceitação e uso ${ }^{24}$, estudos robustos não conseguiram confirmar que o CE contribuiu para a cessação e, mesmo nestes, na generalidade não foi levado em conta o patamar de dependência da nicotina ${ }^{19}$.

A TRN clássica pode apresentar contraindicações em populações específicas, como é o caso dos indivíduos com esquizofrenia (como a eventual potenciação do risco de depressão e suicídio $)^{51}$. Alguns autores consideram que o uso do CE nestes diminui significativamente o consumo de $\mathrm{CCs}^{28,51}$, hábito esse muito prevalente na população psiquiátrica. Para além disso, há quem coloque a possibilidade de os esquizofrénicos consumirem tabaco para atenuar alguns efeitos secundários dos neurolépticos; outros, por sua vez, consideram ser possível que a mesma alteração genética/ bioquímica que pode contribuir para o despoletar desta doença possa, simultaneamente, aumentar a suscetibilidade para o consumo tabágico; aliás, este hábito é muito melhor aceite dentro das instituições psiquiátricas (face a outros estabelecimentos de saúde). Alguns destes indivíduos também não estão interessados em parar o consumo porque acreditam que tal iria piorar a sua situação psiquiátrica ${ }^{51}$.

A prevalência de tabagismo em asmáticos não difere muito da população geral. Uma investigação selecionou um amostra destes indivíduos, que transitaram entre o CC e o CE, tendo verificado melhorias espirométricas, na hiperresponsividade brônquica e no controlo global da asma; daí que estes investigadores tenham considerado que tal era uma opção razoável neste contexto ${ }^{58}$. Contudo, outra investigação avaliou a prevalência de sintomas em estudantes asmáticos consumidores de CE e concluiu que esta era superior à verificada na mesma população não utilizadora do produto, mencionando também um maior absentismo devido a sintomas de maior intensidade50.

A comercialização de CEs nos EUA superou sem qualquer comparação o uso de outros produtos previamente aprovados para cessação tabágica (como a TRN e o Champix, exemplo) $)^{31}$.

Nenhuma das técnicas usadas até agora mimetizava o ato de fumar. Ou seja, mesmo quando a nicotina é administrada via endovenosa, a satisfação não é equivalente a quando é fumada ${ }^{10}$.

Um ano após verificou-se que $6 \%$ dos indivíduos que inicialmente só consumiam o CE voltou ao CC, ainda que $92 \%$ se tenham mantido só com o novo produto ${ }^{19}$.

\section{Gravidez}

Quanto à gravidez e aleitamento, alguns investigadores defendem que não há evidência suficiente relativa ao CE, pelo que o seu uso não deve ser recomendado ${ }^{21}$.

Contudo, a publicidade dá a ideia que este produto é pouco lesivo, pelo que algumas grávidas poderão ponderar consumir tal; para além disso, tem havido aumento da utilização do mesmo em senhoras de idade fértil. Os próprios conviventes da grávida, por vezes, também partilham esta ideia, pelo que deixam de existir entraves sociais para o 
consumo ${ }^{59}$.

\section{Vantagens}

O consumo de CE é mais económico que o $\mathrm{CC}^{33}$, sobretudo quando são utilizadas as recargas $8,21,34,38$; ainda que alguns países (como Portugal) tenham aumentado os impostos destes produto, de forma a o tornar menos aliciante neste contexto, como já se mencionou.

Alguns estudos reportam que o CE diminui o craving e a abstinência, pelo que poderá ser útil na cessação $8,11,13,18,28,60$ ou pelo menos na redução tabágica ${ }^{28}$, como já se referiu. Os consumidores do CE referem menor dependência, ou seja, não sentem um craiving tão intenso como pelo CC; por exemplo, existem relatos a afirmar que quando se esquecem do dispositivo em casa, são capazes de trabalhar normalmente, sem abstinência. Aliás, alguns vão diminuindo a concentração de nicotina e planeiam no futuro vaporizar com soluções sem ela ${ }^{33}$.

Nem os produtos da TRN libertam a nicotina na mesma velocidade ou dose que o CC; ainda assim o mais rápido é o inalador de nicotina, segundo alguns investigadores, mas só está disponível em alguns países. Contudo, nenhum dos produtos da TRN proporciona os comportamentos e sensações diretamente associadas ao ato de fumar, como ocorre com o CE. Ou seja, não só este é fisicamente semelhante, como gera vapor ${ }^{11-13}$ e pode até proporcionar a mesma sensação táctil e/ou ter uma luz na ponta38,40,41. Por isso, alguns investigadores colocaram a hipótese de o CE ser eventualmente mais eficaz na cessação que a TRN clássica ${ }^{11}$ ou, pelo menos, igualmente eficaz ${ }^{28}$. Contudo, o inalador médico de nicotina é considerado mais seguro que o $\mathrm{CE}^{34}$.

Há quem defenda que o CE diminui o consumo dos CCs, sem efeitos secundários significativos $^{12}$. Alguns autores consideram que se o indivíduo não tem capacidade e/ou motivação para parar de fumar deverá, pelo menos, consumir nicotina na forma associada a menos riscos ${ }^{1,60,61}$. O CE contém 500 a 140039 ou 180027 vezes menos nitrosaminas ${ }^{57}$ e 9 a 450 vezes menos carbonilos ${ }^{39}$. Aliás, analisando as concentrações dos principais agentes químicos existentes no $\mathrm{CE}$ com os níveis máximos permitidos para essas substâncias, quando usadas em meio laboral, a situação não é nada preocupante. Para além disso, os níveis para quem estiver próximo (exposição passiva) serão obviamente inferiores ${ }^{57}$.

O CC, mesmo que em consumo diminuto, causa geralmente um atraso no relaxamento do miocárdio, sobretudo a nível do ventrículo esquerdo, ainda no primeiro minuto de consumo; tal não se verificou no $\mathrm{CE}$, mesmo após sete minutos de consumo contínuo. $\mathrm{A}$ explicação fisiopatológica reside no facto de que o $\mathrm{CC}$ fornece radicais livres que promovem o stress oxidativo e a inflamação, logo, com alterações mitocondriais e danos a nível do DNA; a consequência aguda é o atraso no relaxamento do miocárdio e a consequência a longo prazo poderá ser a aterosclerose. A ausência de combustão faz com que não surjam acroleína, acetaldeído e formaldeído (que promovem o stress oxidativo, tendo por isso caraterísticas cardiotóxicas) ${ }^{27}$. Está também descrita a escassez de hidrocarbonetos aromáticos policíclicos, por não existir combustão, tal como ausência28 ou menor concentração de monóxido de carbono ${ }^{17,21,51}$. Também em relação à citotoxicidade miocárdica, um estudo concluiu que os agentes englobados no CE eram lesivos (incluindo os aromas) mas, ainda assim, de forma mais suave que o $\mathrm{CC}^{49}$.

O CE permite uma recuperação do paladar e do olfato, atenua a diminuição da aptidão 
cardiovascular e diminui a tosse e a dispneia ${ }^{32-34}$.

Dentro dos consumidores simultâneos de CE e CC, verificou-se que ocorreu uma diminuição do consumo dos CCs, tal como para a TRN, o que atenua a mortalidade associada $^{19}$. Um estudo quantificou que, após um ano, 46\% dos consumidores em simultâneo de CE e CC, deixou os segundos, mesmo com patamares iniciais de dependência elevada ${ }^{9}$.

A maioria usa concentrações elevadas de nicotina no início mas consegue diminuir com o tempo, ainda que a percentagem dos que usam cartuchos sem nicotina sejabaixa ${ }^{9}$.

O CE não liberta geralmente o aroma desagradável associado ao tabaco ${ }^{34}$ o que, para alguns, a partir de determinada altura, também poderá ser uma vantagem.

\section{Desvantagens}

Os mais jovens são os mais dispostos a experimentar nicotina através de produtos diferentes do $\mathrm{CC}^{2,4,28}$, tal como os indivíduos com maior nível económico ${ }^{28}$, sobretudo se existirem aromas apelativos ${ }^{41}$. Este grupo populacional considera que as formas menos convencionais de consumir nicotina são acessíveis, convenientes, modernas e divertidas; mesmo os não ttabagistas ${ }^{2,36}$. A influência entre pares demonstrou-se aqui bastante importante2, como já se referiu. Ao publicitar a mensagem de que finalmente há uma forma segura (ou, pelo menos, mais suave) de consumir nicotina, tal poderá incentivar a utilização do $C E$ nos mais jovens, mesmo que não consumidores do $C^{4,13,26,28}$; colocando em causa as conquistas das últimas décadas, ou seja, passando a considerarse novamente que fumar é "normal", aceitável e sem riscos relevantes ${ }^{24,37}$.

Devido à ausência de legislação direta, este produto está a ser publicitado em meios proibidos ao CC há muitos anos, como a televisão, cinema, internet e atividades associadas à música e desporto46, como já se desenvolveu neste artigo.

Alguns investigadores, ainda que em minoria, consideram que o CE é mais tóxico devido ao atingimento pulmonar mais direto a nível da nicotina e do propilenoglicol ${ }^{34}$; para além disso, também é mencionado que a libertação de nicotina é desproporcionalmente superior na cavidade oral em relação às vias respiratórias, versus o $\mathrm{CC}^{15}$. Cada aspiração que se faz no CE pode conter cerca de $10^{15}$ moléculas de radicais livres ${ }^{49}$. Outros autores afirmam também que em estudos com roedores encontrou-se diminuição da glutationa, enzima relevante no equilíbrio da oxidação-redução, aumentando o patamar de dano pulmonar ${ }^{21}$.

Para além disso, o ato de recarregar (em caso de derrame) pode implicar intoxicação ${ }^{13,23}$ ou até a absorção de uma dose fatal de nicotina (30 a 60 mg para adultos e cerca de 10 mg paracrianças) ${ }^{34}$. Nos EUA, segundo a organização que apoia as intoxicações, existiram (entre 2010 e 2014) 301 a 512 chamadas mensais associadas ao CE, número esse mais elevado nos meses estivais. A maioria dos casos $(51 \%)$ ocorreu em indivíduos até cinco anos de idade, sendo o segundo grupo etário mais prevalente o da segunda década de vida (42\%). As vias de intoxicação mais frequentes (por ordem decrescente) foram a inalatória, ocular, cutânea e digestiva. O CE justificou 58\% das chamadas associadas à intoxicação por nicotina ${ }^{23}$. Contudo, outros autores também em 2014 assinalam cerca de 3500 eventos em crianças com menos de seis anos (atraídos sobretudo pela cor da embalagem e aromas). Para todas as idades, nos primeiros quatro meses de 2014 foram quantificadas 623 exposições. Estão também descritas explosões e queimaduras faciais e nas mãos, sobretudo devido ao mau funcionamento com dispositivos com baterias de lítio ${ }^{21}$. 
Está descrito que alguns produtos estão rotulados como não tendo nicotina, mas esta foi doseada em algumas marcas ${ }^{17,28}$, tal como já se mencionou.

Para além disso, muitos relatam falhas técnicas do dispositivo com alguma frequência ${ }^{34}$. As empresas que produzem o CE não estão também sujeitas ao mesmo controlo rigoroso que a indústria farmacêutica (produtora da TRN) ${ }^{21,34}$.

De realçar ainda que a aquisição do CE pode ocorrer via internet, sem qualquer controlo etário ${ }^{21}$.

Acreditando que o CE também poderá consolidar ou criar uma dependência de nicotina, alguns autores acreditam que o seu consumo possa facilitar o consumo de outras substâncias psicoativas21. O dispositivo pode mesmo ser adaptado e permitir o consumo de outras substâncias (como a marijuana) ${ }^{34}$.

Alguns autores consideram que o consumo do CE potencia a dependência da nicotina e que não se associa a uma diminuição dos CC utilizados4. Um estudo norte-americano, por exemplo, concluiu que o consumo dos CEs nos jovens desse país associava-se à intenção de continuar a fumar e não de interromper o CC62. Ou seja, o consumo do CE em estudantes não parece contribuir para a interrupção do $\mathrm{CC}^{63}$.

Alguns investigadores defendem que o consumo de CE, mesmo em indivíduos que nunca tenham utilizado o CC, aumenta a probabilidade de o fazerem posteriormente ${ }^{36}$, incluindo adolescentes ${ }^{43}$.

\section{Vantagens ou desvantagens?!}

Algumas condições associadas e este produto podem, simultaneamente, ser consideradas como vantagem ou desvantagem, consoante o ângulo de análise. Por exemplo, muitos estudos referem a diferente (e eventualmente má) qualidade entre marcas, pelo que a libertação de nicotina não é homogénea entre modelos ou utilizadores ${ }^{13,18,38}$, nem constante ao longo do consumo de cada embalagem ${ }^{13,38}$; ou seja, diminui bastante depois de 150 a 180 puffs38. Consumidores mais inexperientes absorvem menos nicotina15,18,33,38,61; ainda assim, considera-se que o nível de nicotina é substancialmente inferior ao existente no CC. À medida que o CE é consumido, é necessária uma força de sucção cada vez maior, uma vez que a quantidade de aerossol produzida é progressivamente menor, sobretudo a partir do décimo puff, sensivelmente 42 .

Os efeitos secundários costumam ser discretos e pouco relevantes9. Num estudo, a maioria relatou com maior frequência a existência de secura na boca/ garganta e tosse seca $^{12,51}(32,4 \% \text { para ambas })^{32}$, secundária ao propilenoglicol ${ }^{9,12,51}$ e ao glicerol $^{9}$. Outros efeitos secundários descritos são o surgimento/ agravamento de patologia respiratória e/ou cardíaca, desorientação, convulsões, hipotensão, queimaduras faciais, alterações visuais $^{20}$, cefaleias, náusea/ vómito ${ }^{12,20,51}$, tontura, dor abdominal ${ }^{20}$, ulceras ${ }^{12,51} \mathrm{e}$ sonolência ${ }^{20}$. Em 3,5\% verificou-se a ocorrência de hipertensão arterial, geralmente associada a palpitações e taquicardia ${ }^{30}$.

Em algumas marcas de CEs foram encontrados fármacos da medicina convencional (como o rimonaban ou o tadalafil) ${ }^{16,28}$, que inibe o apetite para a ingestão de alimentos e potencia o desempenho sexual, respetivamente; aliás, não é tecnicamente difícil adicionar qualquer substância ao cartucho ${ }^{28}$, como já se mencionou.

As partículas produzidas pelos CEs variam (em dimensão) entre os 100 e os 600 nanómetros; apesar de ser possível que partículas menores se agreguem, não é usual que 
este valor máximo seja ultrapassado; ainda assim, acredita-se que algumas consigam se depositar nos alvéolos. Apesar de se absorver menos nicotina com o CE e de o processo ser mais lento, alguns fumadores reportam que conseguem permanecer mais tempo sem sentir a necessidade de fumar novamente ${ }^{61}$. Contudo, simultaneamente, outros também afirmam que necessitam de inalar mais profundamente ${ }^{48}$.

Para além disso, a própria velocidade de absorção de nicotina, mesmo com CEs de última geração é menor que no $\mathrm{CC}^{27}$. Com o CC a nicotina demora cerca de vinte segundos a iniciar os seus efeitos; via CE e TRN tal ocorrerá apenas em alguns minutos. Logo alguns investigadores consideram que o potencial de adição é menor. Contudo, é de esperar que a indústria tente aumentar a libertação/ absorção de nicotina, para mimetizar ainda mais o $\mathrm{CC}^{28}$.

Um estudo norte-americano quantificou que $77 \%$ dos utilizadores do CE achavam-no menos satisfatório que o CC, sendo que alguns apenas o mantinham por o acharem menos tóxico ${ }^{21}$. Outro estudo quantificou que cerca de $52 \%$ dos consumidores do CC também usavam o CE; contudo, investigações prévias apenas registaram valores entre 3,4 e 12,7\%4. Em 2014 a prevalência do consumo do CE diária nos alunos dos 8, 9 e $12^{\circ}$ anos nos EUA foi de 8,7-16,2 e 17,1\%, respetivamente; versus 4- 7,2 e 13,6 para o CC 62 .

Os CEs emitem aldeídos em concentração significativa, secundários à decomposição térmica de alguns agentes químicos (como o glicerol e o propilenoglicol), mas apenas nos "puffs secos"- nestas circunstâncias fica um sabor muito forte secundário ao aquecimento excessivo; estes, por serem desagradáveis, são evitados por si só. Ou seja, no uso normal do CE as emissões de aldeídos são mínimas, com dispositivos de última geração. Estes eventos dependem da potência do aparelho, ainda que de forma não linear. Para além disso, como só é detetado pelo sabor, não são registáveis na experimentação laboratorial em não humanos. Os dispositivos mais recentes apresentam material transparente a revestir o líquido, de forma ao indivíduo perceber quando este fica diminuto para continuar a utilizar de forma confortável. Alguns autores também descrevem que este fenómeno é mais frequente nos aparelhos novos- globalmente estima-se que tal possa ocorrer em média uma vez por dia. Acredita-se que alterando a composição dos líquidos, para futuro, se possa diminuir eventualmente a produção de aldeídos ${ }^{64}$.

A nível laboral estão descritos casos em que um funcionário alicia colegas e/ou a chefia a experimentar e, dado verificarem que podem teoricamente consumir sem abandonar o posto de trabalho e sem o tornar o ambiente interno percetivelmente mais desagradável, vários passaram a ser consumidores de $\mathrm{CE}^{33}$.

\section{Legislação}

A maioria dos países não tem qualquer legislação relativa ao CE. Contudo, em função dos eventuais riscos associados para a saúde pública, alguns países ponderam criar tal a curto ou médio prazos. No entanto, dentro de um mesmo país, podem encontrar-se assimetrias, como é o caso dos diferentes estados norte-americanos ${ }^{22}$.

Os produtores comercializam o CE como terapêutica para diminuir e/ ou interromper o consumo do CC, mas tal não foi reconhecido pela FDA ${ }^{23}$. Aliás esta instituição e a OMS reconheceram que o seu consumo deveria ser evitado (independentemente do objetivo) ${ }^{27}$; estas duas instituições proíbem a declaração de serem produtos terapêuticos ${ }^{9}$. Em maio de 2016 a FDA esclareceu que todos os produtos com tabaco estavam sob o seu controlo, incluindo o $C E^{14,21}$, ainda que não existam efeitos práticos imediatos, porque as empresas 
da área pediram um tempo para adaptação. Por exemplo, até agora o produto não tinha de ser oficialmente testado antes de ser colocado no mercado ${ }^{21}$.

Em países como o Brasil, Singapura, Canadá, Seychelles e Uruguai, a venda de CEs está proibida. Já nos EUA, a FDA ainda não assumiu nenhuma posição oficial, ainda que alguns estados tenham restrito a venda a maiores de idade e/ou proibido o consumo nos mesmos locais que o $\mathrm{CC}^{19}$.

A CEE pretendeu em 2014 regular os CEs que fornecessem uma concentração de nicotina superior ou igual a $20 \mathrm{mg} / \mathrm{ml}$ (equivalente a um maço de cigarros), surgiram diretrizes também para que os produtos fossem à prova do consumo infantil e que as embalagens deveriam ser claras em relação à composição e eventuais efeitos secundários. Definiu também que os cartuchos recarregáveis não deveriam ultrapassar os dois mililitros. Quanto à publicidade, adotou as mesmas regras em vigor para o CC. Já no Reino Unido, por sua vez, o produto foi classificado como auxiliar terapêutico para os tabagistas que pretendam reduzir os danos e/ou parar ${ }^{19}$. Contudo, em 2015 foi proibida a venda a menores de idade e exigido um embalamento à prova de abertura por crianças ${ }^{21}$.

\section{CONCLUSÕES}

Com uma frequência cada vez maior, os fumadores questionam os profissionais de saúde acerca do CE, dentro e fora do contexto laboral; uns porque já o consomem, outros porque estão a pensar fazê-lo. É necessário, por isso, estar a par das conclusões dos estudos já elaborados sobre o tema. Globalmente considera-se que a publicidade é enganosa, ou seja, o ato não é isento de riscos, nem está provado, com evidência científica, que contribua, neste momento, para a cessação do consumo da nicotina. Contudo, simultaneamente, também parece claro que os agentes químicos envolvidos estão em muito menor número e concentração que no cigarro convencional, pelo que parece ser uma alternativa menos tóxica de consumir nicotina. Para além disso, o CE parece ajudar alguns indivíduos a interromper ou reduzir os CCs e, simultaneamente, alguns referem sentir uma menor adição. No entanto, passar tal mensagem também pode contribuir para a diminuição da motivação para realizar a cessação tabágica ou pode até aliciar nãotabagistas ao início do consumo, comprometendo os ganhos obtidos na luta contra o tabaco das últimas décadas. A segurança dos agentes químicos que são introduzidos nos locais onde este produto é consumido ainda não foi comprovada com clareza, nem se encontram estudos específicos para o contexto laboral, ainda que a análise de qualquer ambiente fechado possa generalizar-se para a maioria dos ambientes ocupacionais, sobretudo se não existirem áreas restritas, temperaturas extremas e/ou a presença de outros agentes químicos ocupacionais. O consumo do CE em meio laboral deve ser desaconselhado e as restrições existentes para o CC também devem ser aplicadas ao CE.

\section{CONFLITOS DE INTERESSE}

Não se aplicam.

\section{AGRADECIMENTOS}

Não se aplicam.

\section{BIBLIOGRAFIA}

1-Popova L, Ling P. Alternative tobacco product use and smoking cessation: a national study. American Journal of Public Health. 2013, e1-e7. 
2- Choi K, Fabian I, Mottey N, Corbett A, Forster J. Young adult's favorable perceptions of snus, dissolvable tobacco products and electronic cigarettes: findings from a focus group study. American Journal of Public Health. 2012, 102 (11), 2088-2093.

3- Hu S, Neff L, Agaku I, Cox S, Day H, Holder-Hayes E et al. Tobacco products use among adults- United States, 2013-2014. Centers for Disease Control and PreventionMMWR. 2016, 65(27), 1-8.

4- Levault K, Muella-Luckey G, Waters E, Fogleman A, Crumly D, Jenkings W. E-cigarettes: who's using them and why? The Journal of Family Practice. 2016, 65(6), 390-397.

5- Singh T, Arrazola R, Corey C, Husten C, Nelf L, Homa D et al. Tobacco use among middle and high school students- United States, 2011-2015. Centers for Diseases Control and Prevention- MMWR. 2016, 65(4), 361-367.

6- Goniewick M, Lingas E, Hajek P. Patterns of electronic cigarette use and user beliefs about their safety and benefits: an internet survey. Drug and Alcohol Review. 2013, 32, 133-140.

7- Mcauley T, Hopke P, Zhao J, Babaian S. Comparison of the effects of e-cigarette vapor and cigarette smoke on indoor air quality. Inhalation Toxicology. 2012, 24(12), 850-857.

8- Czoli C, Hammond D, White C. Electronic cigarettes in Canada: prevalence of use and perceptions among youth and young adults. Canadian Journal of Public Health. 2014, 105(2), e97-e102.

9- Farsalinos K, Romagna G, Tsiapras D, Kyrzopoulos S, Voudris V. Characteristics, perceived side effects and benefits of electronic cigarette use: a worldwide survey of more than 19000 consumers. International Journal of Environmental Research and Public Health. 2014, 4356-4373.

10- McRobbie $\mathrm{H}$, Bullen $\mathrm{C}$, Hajek P. Electronic cigarettes for smoking cessation and reduction (protocol). The Cochrane Library. 2012, 11, 1-8.

11-Bullen C, Williman J, Howe C, Laugesen M, McRobbie H, Parag V et al. Study protocol for a randomized controlled trial of Electronic Cigarette versus nicotine patch for smoking cessation. BiomedCentral. 2013, 13(210), 1-8.

12- Polosa R, Caponnetto P, Morjaria J, Papale G, Campagna D, Russo C. Effect on an electronic nicotine delivery device (e-cigarette) on smoking reduction and cessation: a prospective 6-month pilot study. BMC Public Health. 2011, 11(786), 1-12.

13-Pokhrel P, Fagan P, Little M, Kawamoto C, Herzog T. Smokers try e-cigarettes to quit smoking: findings from a multiethnic study in Hawaii. American Journal of Public Health. 2013, 103(9), e57-e63.

14- Syamll G, Jamal A, King B, Mazurek J. Electronic cigarette use among working adultsUnited States, 2014. Centers for Disease control and Prevention- MMWR. 2016, 65(22), 16.

15- Vansickel A, Weaver M, Eissenberg T. Clinical Laboratory assessment of the abuse liability of an electronic cigarette. Addiction. 2012, 107, 1493-1500.

16- Etter J, Bullen C. Electronic cigarette: users profile, utilization, satisfaction and perceived efficacy. Addiction. 2011, 106, 2017-2028.

17-Yamin C, Bitton A, Bates D. E-cigarettes: a rapidly growing internet phenomenon. Annals of Internal Medicine. 2010, 153(9), 607-609.

18-Dawkins L, Turner J, Roberts A, Soar K. "Vaping" profiles and preferences: an on line survey of electronic cigarette users. Addiction. 2013, 108, 1115-1125.

19- Grana R, Benowitz N, Glantz S. E-cigarettes- a scientific review. Circulation. 2014, 
1971-1985.

1-Popova L, Ling P. Alternative tobacco product use and smoking cessation: a national study. American Journal of Public Health. 2013, e1-e7.

20- Chen L. FDA summary of adverse events on electronic cigarettes. Nicotine \& Tobacco research, 15(2). 2013, 615-616.

21- Smith L, Brar K, Srinivasan K, Enja M, Lippmann S. E-cigarettes: how "safe" are they? The Journal of Family Practice, 65(6), 380-385.

22- (sem autor). Restrictions on use of e-tobacco aim to limit addiction risk. Primary Health Care. 2014, 24(4),7-8.

23- Chatham-Stephens K, Law R, Taylor E, Melstrom P, Bunnel R, Wang B et al. Calls to Poison Centers for exposures to Electronic Cigarettes- United States, September 2010February 2014. MMWR. 2014, 63(13), 292-293.

24-Trueland J. A burning issue. Nursing Standard. 2013, 28(18), 28-30.

25-Schripp T, Markewitz P, Uhde E, Salthammer T. Does e-cigarette consumption cause passive vaping? Indoor Air. 2013, 23, 25-31.

26- Choi K, Forster J. Characteristics associated with awareness, perceptions and use of electronic nicotine delivery systems among young US Midwestern adults. American Journal of Public Health. 2013, 103(3), 556-561.

27- Farsalinos K, Tsiapras D, Kyrzopoulos S, Sauvopoulou M, Voudris V. Acute effects of using electronic nicotine-delivery device (electronic cigarette) on myocardial function: comparison with the effects of regular cigarettes. BMC Cardiovascular Disorders, BioMed Central. 2014, 14(78), 1-17.

28- Nowak D, Jorres R, Ruther T. E-cigarettes- prevention, pulmonary health and addiction. Deuches Arzteblatt International. 2014, 111, 349-355.

29- Farsalinos K, Romagna G, Tsiapras D, Kyrzopoulos S, Spyrou A, Voudris V. Impact of flavor variability on electronic cigarette use experience: an internet survey. International Journal of Environmental Research and Public Health. 2013, 10, 7272-7282.

30- Hua M, Alfi M, Talbot P. Health-related effects reported by electronic cigarette users in online forums. Journal of Medical Internet Research. 2013, 15(4), e59, 1-9.

31- Vansickel A, Weaver M, Eissenberg T. Clinical laboratory assessment of the abuse liability of electronic cigarette. Addiction, 107, 1493-1500.

32- Richardson A, Xiao H, Vallone D. Primary and dual users of cigars and Canadian Agency for Drugs and Technologies in Health. Electronic cigarettes: a review of the clinical evidence and Safety. 2012, 1-25.

33- McQueen A, Tower S, Summer W. Interviews with "vapers", implications for future research with electronic cigarettes. Nicotine \& Tobacco Research. 2011, 13(9), 860-867.

34- Etter J. Electronic cigarettes: a survey of users. BMC Public Health. 2010, 10(231), 1-7. 35- Wang M, Wang J, Cao J, Wang H, Hu R. Cigarette smoking and Electronic cigarettes use: a meta-analysis. International Journal of Environmental Research and Public Health. 2016, 13(120), 1-16.

36- Primack B, Soneji S, Stoolmiller M, Fine M, Sargent J. Progression to traditional cigarette smoking after electronic cigarette use among US adolescents and young adults. JAMA Pediatrics. 2015, 169(11).

37- Voigt K. Smoking norms and the regulation of e-cigarettes. American Journal of Public Health. 2015, 105(10), 1967-1972.

38-Goniewicz M, Kuma J, Gawron M, Knysak J, Kosmider I. Nicotine levels in electronic 
cigarettes. Nicotine \& Tobacco Research. 2013, 15(1), 158-165.

39-Farsalinos K, Romagna G, Tsiapras D, Kyrzopoulos S, Voudris V. Evaluation of Electronic Cigarettes Use (vaping) topography and estimation of liquid consumption implications for research protocol standards definition and for public health authorities regulation. International Journal of Environmental Health. 2013, 10, 2500-2514.

40-Hodje J, Collmer V, Orenstein D, Millea C, Buren L. Reconsidering the legality of cigarette smoking- advertising on television, Public Health and the Law. Journal of Law, Medicine and Ethics. 2013, 369-373.

41-Wellscheid K, Kremzer M. Electronic cigarettes: safety concerns and regularity issues. American Journal Health System Pharmacology. 2009, 66, 1740-1742.

42-Trtchounian A, Williams M, Talbot P. Conventional and electronic cigarette (ecigarettes) have different smoking characteristics. Nicotine \& Tobacco Research. 2010, 12(9), 905-912.

43- Leventhal A, Strong D, Kirkpatrick M, Unger J, Sussman S, Riggs N et al. Assotiation of Electronic Cigarette use with initiation of combustible tobacco product smoking in early adolescence. JAMA. 2015, 314(7), 700-707.

44- Wang L, Zhan Y, Li Q, Zeng D, Leischow S, Okamoto J. An examination of electronic cigarette content on Social Media: Analysis of E-cigarette flavor content on Reddit. International Journal of Environmental Research Public Health. 2015, 12, 14933-14952.

45- Vansickel A, Eissenberg T. Electronic cigarettes: effective nicotine delivery after acute administration. Nicotine \& Tobacco Research. 2013, 15(1), 267-270.

46- Collins A. Vape me out to the ball game! Journal of Environmental Health. 2013, 76(5), 6-7.

47- Ingebrethsen B, Cole S, Alderman S. Electronic cigarette aerosol particle size distribution measurements. Inhalation Toxicology. 2012, 24(14), 976-984.

48- Williams M, Talbot P. Variability among electronic cigarettes in the pressure drop, airflow rate and aerosol production. Nicotine \& Tobacco Research. 2011, 13(12), 12761283.

49- Farsalinos K, Romagna G, Allifranchini E, Ripamonti E, Bocchietto E, Todeschi S et all. Comparison of the cytotoxic potential of cigarette smoke and electronic cigarette vapour extract on cultured myocardial cells. International Journal of Environmental Research and Public Health. 2013, 10, 5146-5162.

50- Cho J, Paik S. Association between Electronic Cigarette use and Asthma among high school students in South Korea. PLOS ONE. 2016, march 4, 1-13.

51- Caponnetto O, Auditore R, Russo C, Cappello G, Polosa R. Impact of an Electronic cigarette smoking reduction and cessation in schizophrenic smokers: a prospective 12months pilot study. International Journal of Environmental Research and Public Health. 2013, 10, 446-461.

52- Farsalinos K, Gillman G, Poulas K, Voudris V. Tobacco-specific nitrosamines in electronic cigarettes: comparison between liquid and aerosol levels. Experimental Research and Public Health. 2015, 12, 9046-9053.

53- Farsalinos K, Voudris V, Poulas K. Are metals froms electronic cigarettes a reason for health concern? A risks assessment analysis of currently available literature. 2015, 12, 5215-5232.

54- Biener L, Song E, Sutfin E, Spangler J, Wolfson M. Electronic Cigarette Trial and Use among young adults: reasons for trial and cessation of vaping. International Journal of 
environmental Research and Public Health. 2015, 12, 16019-16026.

55- Chu K, Unger J, Allem J, Pattarroyo M, Soto D, Cruz T et al. Diffusion of messages from electronic cigarette brand to potential users through Twitter. PLOS ONE. 2015, December 18, 1-11.

56- Dawkins L, Turner J, Crowe E. Nicotine derived from the electronic cigarette improves time-based prospective memory in abstinent smokers. Psychopharmacology. 2013, 227, 377-384.

57- Burstyn I. Peering through the mist: systematic reviews of what the chemistry of contaminants in electronic cigarettes tell us about health risks. BMC Public Health- Biomed Central. 2014, 1-27.

58- Polosa R, Morjaria J, Caponetto P, Caruso M, Stano S, Battaglia E et al. Effect of smoking abstinence and reduction in asthmatic smokers switching to electronic cigarettes: evidence for harm reversal. International Journal of Environmental Research and Public Health. 2014, 11, 4965-4977.

59- Kahr M, Padgett S, Shqe C, Griffin E, Xie S, Gonzalez P et al. A qualitative assessment of the perceived risks of electronic cigarette and hookah use in pregnancy. BMB Public Health. 2015, 13(120), 1-16.

60- Zyioud S, Al-Jabi S, Sweilen W. Wordwide research productivity in the field of the electronic cigarettes: a bibliometric analyze. BMC Public Health, Biomed Central. 2014, 14, 1-16.

61-Zhang Y, Summer W, Chen D. In vitro particle size distributions in electronic and conventional cigarette aerosols suggest comparable deposition patterns. Nicotine \& Tobacco Research. 2012, 15(2), 501-508.

62- Park J, Seo D, Lin H. E-cigarette use and intention to initiate or quit smoking among youth. American Journal of Public Health. 2016, 106(4), 672-678.

63- Sutfin E, Reboussin B, Debinski B, Wagoner K, Spangler J, Wolfson M. The impact of trying electronic cigarettes on cigarette smoking by college students: a prospective analyses. American Journal of Public Health. 2015, 105(8), e83-e89.

64- Farsalinos K, Voudris V, Poulas K. E-cigarettes generate high levels oF aldehydes only in "dry puff" conditions. Addiction. 2015, 110, 1352-1356.

Fluxograma: Caraterização da Pesquisa

s_1

(1) Mónica Santos

Licenciada em Medicina; Especialista em Medicina Geral e Familiar; Mestre em Ciências do Desporto; Especialista em Medicina do Trabalho; Presentemente a exercer nas empresas Medicisforma, Clinae, Servinecra e Serviço Intermédico; Diretora Clínica da empresa Quercia; Diretora da Revista Portuguesa de Saúde Ocupacional on line; Endereços para correspondência: Rua Agostinho Fernando Oliveira Guedes, 42 4420-009 Gondomar; s_monica_santos@hotmail.com.

(2) Armando Almeida 
Mestre em Enfermagem Avançada; Especialista em Enfermagem Comunitária; Pósgraduado em Supervisão Clínica e em Sistemas de Informação em Enfermagem; Docente na Escola de Enfermagem (Porto), Instituto da Ciências da Saúde da Universidade Católica Portuguesa; Diretor Adjunto da Revista Portuguesa de Saúde Ocupacional on line; aalmeida@porto.ucp.pt. 